\title{
Erratum: Water-based Peeling of Thin Hydrophobic Films [Phys. Rev. Lett. 119, 154502 (2017)]
}

\author{
Sepideh Khodaparast, François Boulogne, Christophe Poulard, and Howard A. Stone
}

(Received 10 December 2018; published 28 December 2018)

DOI: 10.1103/PhysRevLett.121.269901

There is a misprint in the axis of panel (d) in Fig. 3. Following the data in Fig. 3(c), the corresponding horizontal axis in Fig. 3(d) should have units of $\mathrm{mN} / \mathrm{m}$. The corrected graph is presented below. We note that the corresponding statement about the limit of viscous dissipation (on page 4 of the Letter) should read "we note that the critical transition, peeling to no peeling, occurs when $\eta U / \gamma=C a=O\left(10^{-4}-10^{-3}\right)$ ". We thank Jingcheng Ma of the University of Illinois at Urbana-Champaign for identifying this misprint.

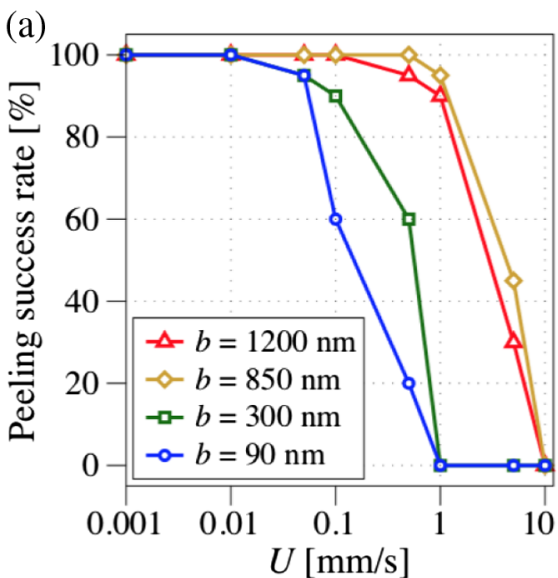

(b)
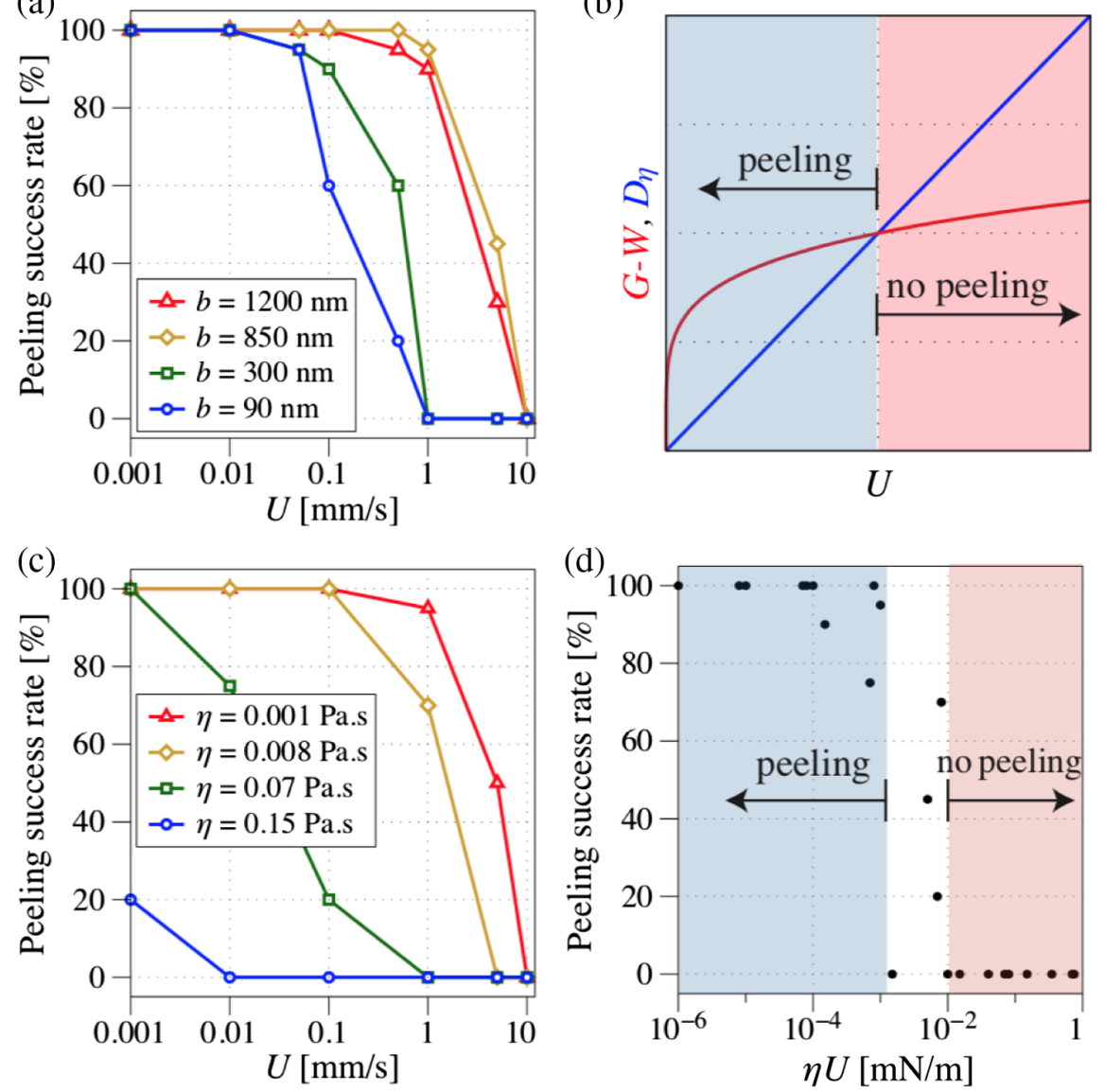

FIG. 3. Role of viscous dissipation $D_{\eta}$. (a) Capillary peeling becomes less efficient at higher speeds $U>1 \mathrm{~mm} / \mathrm{s}$, especially for very thin films $b<100 \mathrm{~nm}$. The success rate is calculated for capillary peeling performed on 20 samples. (b) Velocity dependence of the adhesion energy $G$ and the viscous dissipation $D_{\eta}$. The blue domain corresponds to an energetically favorable peeling. (c) Capillary peeling becomes less efficient at higher viscosities $\eta \approx 10^{-2}$ Pa for $b=850 \mathrm{~nm}$. (d) The data from panel (c) collapse when plotted as a function of $\eta U$. Capillary peeling tends to fail for $\eta U>10^{-2} \mathrm{mN} / \mathrm{m}$. 